A. Weisbecker, M. Burmester \& A. Schmidt (Hrsg.): Mensch und Computer 2015

Workshopband, Stuttgart: Oldenbourg Wissenschaftsverlag, 2015, S. 51-59.

\title{
Ein Interaktionskonzept zur Simulation und Analyse von MANV-Einsätzen
}

\author{
Johannes Sautter ${ }^{1}$, Lars Böspflug ${ }^{1}$, Friederike Schneider ${ }^{2}$
}

Fraunhofer-Institut für Arbeitswirtschaft und Organisation IAO ${ }^{1}$

Deutsches Rotes Kreuz - Generalsekretariat ${ }^{2}$

\section{Zusammenfassung}

Planungen für den Massenanfall von Verletzten (MANV) finden bisher hauptsächlich in Form lokaler Einsatzkonzepte statt. Ersteintreffende Führungskräfte sowie medizinische Einsatzleiter entscheiden im Ernstfall ad-hoc und unter Unsicherheit über taktische Kernpunkte der Einsatzbewältigung. Eine lokalspezifische Ressourcenplanung für MANV-Ereignisse unterstützt durch ein computergestützte Modellierungs- und Simulationssystem kann helfen durch lokale Handlungsleitfäden für Einsatzleiter Unsicherheiten zu verringern. Der Artikel beschreibt die iterative Entwicklung eines Interaktionskonzeptes, das durch Fachanwender bedient werden kann, sowie dabei aufgetretene Herausforderungen wie eine selbstbeschreibungsfähige Interaktionsgestaltung und das Vertrauen der Anwender in die Validität der Simulationsergebnisse. Der Grad der Interaktivität der Benutzer-ModellInteraktion sowie die Anzahl betrachteter Ergebnis-Indikatoren variieren hierbei.

\section{$1 \quad$ Einleitung}

Im medizinischen Bevölkerungsschutz agieren haupt- und ehrenamtliche Kräfte von Hilfsorganisationen wie Malteser, Deutsches Rotes Kreuz, Arbeiter-Samariter-Bund und Johanniter im Auftrag des Bundes, der Länder und der Kommunen. Neben der medizinischen Versorgung im Regelrettungsdienst gewährleisten sie auch die Versorgung im Fall von Großschadenslagen. Ein Massenanfall von Verletzten (MANV) ist dabei definiert als ein Notfall mit einer größeren Anzahl von Verletzten, Erkrankten oder Betroffenen, der mit vorhandenen Ressourcen aus dem Rettungsdienstbereich nicht bewältigt werden kann (BBK 2013). Als ein wesentlicher Prozessschritt des MANV weist die Sichtung Patienten Sichtungskategorien (SK) zu. Rot steht hierbei für Schwerverletzte der Sichtungskategorie 1, gelb für mittelschwer-Verletzte (SK2) und grün für Leichtverletzte (SK3). Praktiker und Experten der Hilfsorganisationen wünschen sich aktuell eine bessere Ressourcenplanung über Kommunen- und Landkreisgrenzen hinweg (DRK 2012). Ein Analyse-Simulationstool kann das regelmäßige Üben von MANV-Einsätzen ergänzen und Einsatzleitern erlauben, 
verschiedene einsatztaktische Optionen unter lokalen Vorgaben zu Rettungsmitteln und Krankenhäusern zu erörtern - ohne gleichzeitig hohe finanzielle Mittel aufwenden zu müssen (Sautter et al. 2014a).

Als potentielle Benutzer eines Analyse-Simulationssystems im besonders sicherheitskritischen Nutzungskontext der Zivilen Sicherheit wurden in einer Kontextanalyse Einsatzleiter und Führungskräfte (u.a. Organisatorischer Leiter Rettungsdienst, Leitender Notarzt) identifiziert. Demnach verfügen sie über mittelmäßige IT-Anwenderkenntnisse und würden das Simulationstool in einer ruhigen unterbrechungsfreien Umgebung anwenden. Weiterhin ist eine Nutzung zu Demonstrationszwecken bei der Führungskräfteausbildung, im Einsatzleitwagen bei Übungen sowie eventuell bei realen Einsätzen möglich. Neben der Bedienung per Maus ist die Benutzung per Touchscreen für den mobilen Einsatz sowie in der Freizeit der Anwender (ggf. abends auf dem Sofa) ebenfalls gewünscht.

Simulationstools werden bisher meist durch Fachanwender und Simulationsexperten bedient. Laien im Bereich Modellierung und Simulation sind durch ihre mangelnden ModellKenntnisse nur schwer in der Lage computergestützte Modelle richtig zu bedienen und vor allem zu interpretieren (Brailsford et al. 2009). Eine Herausforderung für Entwickler ist es daher Systeme so zu konzipieren, dass diese nur valide Parametereingaben zulassen und Ergebnisse adäquat darstellen, so dass auch Laien sie richtig deuten können. Dafür ist innerhalb der Analyse und Konzeptionsphase eine enge Abstimmung zwischen Simulationsmodell und Mensch-Maschine-Schnittstelle notwendig. Aber auch Unsicherheiten des Modells, Basisdaten, Validität und die Akzeptanz bei Nutzern müssen berücksichtigt werden. Über einen Zeitraum von ca. zwei Jahren wurden drei verschiedene Interaktionskonzepte unter der Beachtung bekannter Vorbedingungen konzipiert und mit Benutzern evaluiert. Der vorliegende Artikel beschreibt die Konzeption und Evaluation dieser Interaktionskonzepte. Dabei werden zunächst Parameter und Indikatoren als Grundkonzepte von Analyse-Simulationsmodellen betrachtet. Anschließend wird auf den eigentlichen Prozess der Entwicklung eingegangen.

\section{Grundkonzepte der Simulation für Analysezwecke}

Ein Simulationsmodell wird im Folgenden als eine abstrakte computergestützte Repräsentation eines Systems oder eines Prozesses verstanden (Carson 2005). Simulation bedeutet hierbei ein Modell mit passenden Parametern zu steuern und dessen Ausgaben zu beobachten. Damit die Ergebnisdatensätze eines Analyse-Simulationstools interpretiert werden können, bedarf es quantitativer Indikatoren. Sie sind ein Grundkonzept im Bereich der Modellierung und Simulation und bilden Modellzustände bzw. komplexe Datenreihen und Wertemengen auf einzelne Wertkennzahlen ab (Dihé et al. 2013). Die Nutzung von Indikatoren erlaubt neben dem detaillierten Einblick in die Simulationsergebnisse auch eine aggregierte Analyse. Vor allem der Vergleich unterschiedlicher Simulationsläufe mit unterschiedlichen Analysefragestellungen wird dadurch vereinfacht. Ein Simulationslauf zeichnet sich dabei durch einen bestimmten Anfangs- und Endzustand aus. Ein allgemeines Interaktionskonzept für Analyse-Simulation ist in der Simulationsplattform Netlogo umgesetzt 
und besteht aus der Parametereingabe, die den ersten Schritt der Nutzerinteraktion darstellt. Sobald die Modellausführung gestartet wird, ändert sich die grafische Modellrepräsentation und die Indikatoren-Anzeige (Tisue \& Wilensky 2004).

In einem Workshop mit Anwendern des MANV-Simulationstools konnten mittels eines Brainstormings wesentliche Faktoren eines MANV-Einsatzes identifiziert und priorisiert werden. Die Parameter und Indikatoren aus den einzelnen Kategorien werden im Folgenden aufgelistet:

- Lage: Anzahl Patienten, Wetterverhältnisse, Anzahl der Patienten, die laufen können (Szenario-Parameter)

- Einsatztaktik: Anzahl beteiligter medizinischer Einsatzkräfte, Anzahl verwendeter Einsatzabschnitte, Zeit bis zur Nachalarmierung (Parameter)

- Erfolgskriterien: Zeit bis Vorsichtung abgeschlossen ist, Zeit bis der erste rote Patient behandelt wird, Zeit bis der letzte rote Patient behandelt wurde, Zeit bis alle rot-gesichteten Patienten den Notfallort verlassen haben, Zeit bis letzter rote Patient im Krankenhaus eintrifft (Einsatzziel), Verhältnis zwischen Einsatzkräften und Patienten, Zeit bis RMHP eingerichtet ist, Zeit bis Bereitstellungsraum eingerichtet ist, Zeit bis BHP eingerichtet ist, Zeit bis der letzte Patient im Krankenhaus eintrifft (Indikatoren)

Die zur Verfügung stehende Datengrundlage ist eine wesentliche Vorbedingung zur Entwicklung eines Simulationsmodells. Eine Kalibrierung und Validierung unter Berücksichtigung der zu beantwortenden Analysefragestellung erfolgt als wesentlicher Bestandteil der Modellentwicklung (Railsback \& Grimm 2012). Vertrauen in die Validität des Simulationstools ist Grundbedingung für die Akzeptanz des Systems. Bei der Simulation von MANV-Einsätzen spielen Evaluationsdaten aus realen Einsatzübungen eine wesentliche Rolle (Sautter et al. 2014b). Daten der Leitstelle von vergangenen Realeinsätzen sind weiterhin potentiell nutzbar.

\section{Indikator-Timeline mit vordefinierter Strategie}

Parameter und Indikatoren: Zentrales Element der ersten Iteration in der Entwicklung ist die Visualisierung des einzelnen Indikators ,Zeit bis letzter roter Patient von der Einsatzstelle abtransportiert wurde" auf einer Timeline. Zur Eingabe der Parameter werden dem Nutzer zwei Möglichkeiten zur Verfügung gestellt, um die Einsatzplanung für einen MANV mithilfe der lokal zur Verfügung stehenden Ressourcen handlungsschnell und zielgerichtet durchzuführen: Entweder kann durch die Auswahl eines Einsatzstichwortes der Alarm- und Ausrückeordnung AAO (bspw. „MANV25“) die Ressourcenanforderung definiert werden (,Automatic Deployment“ oben links in Abb. 1) oder es kann eine freie Auswahl der Rettungsmittel erfolgen (links in Abb. 1).

Interaktion: Als wesentliche Bedienelemente stehen dem Nutzer ein Drop-Down-Menü zur Auswahl von Einsatzstichworten der lokalen AAO (siehe oben links in Abb. 1) und eine Zeitleiste, auf der der Indikatorwert abgelesen werden kann, zur Verfügung. Des Weiteren 
können verschiedene Einsatzfahrzeuge manuell per Drag'n Drop hinzugefügt und weggenommen werden. Durch die schnelle Auswirkung der Eingaben auf das Modell ergibt sich eine sehr hohe Interaktivität mit vordefinierten Strategien zum Wählen und Ablesen.

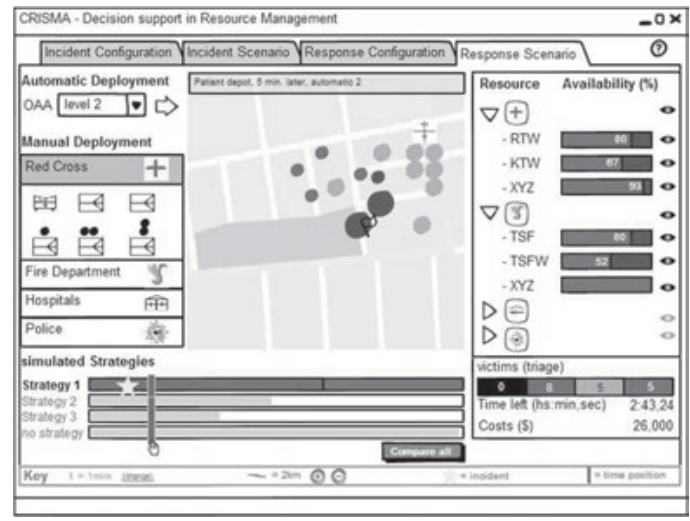

Abbildung 1: Konzept der Indikator-Timeline mit vordefinierter Strategie

Zuvor werden taktische Einsatzabläufe als Strategien durch den Benutzer festgelegt. Die Konsequenzen der ausgewählten Strategie werden auf Basis des zentralen Indikators ausgewertet. Dies erlaubt es dem Benutzer, eine schnelle Einschätzung zur Eignung der gewählten Parameter $\mathrm{zu}$ bekommen. Dennoch können die Ergebnisse anderer Bewertungsindikatoren wie beispielsweise die Anzahl der Verletzten einer Sichtungskategorie zu einem Zeitpunkt für jede Strategie explorativ per Klick auf die Timeline dargestellt werden. Die Anzeige der Anzahl der Verletzten an der Schadensstelle (unten rechts in Abb. 1) wird dann zum ausgewählten Zeitpunkt angezeigt. Neben den Verletztenstatus zeigt Abb. 1 alle zur Verfügung stehenden Ressourcen einer Region (rechts in Abb. 1) sowie eine Kartendarstellung des Einsatzgebiets (Mitte in Abb. 1).

Evaluation: Eine Evaluation des Konzeptes fand im Rahmen eines Poster-Workshops und eines User-Guided Walkthroughs statt. Die Teilnehmer des Poster-Workshops waren Experten des Bevölkerungsschutzes. Hierbei wurde identifiziert, dass es möglich sein könnte, jedem Einsatzabschnitt (bspw. Patientenablage) implizit eine fachliche Einsatztaktik/Strategie zuzuordnen. Außerdem erkannten die Nutzer nicht, dass das Hauptergebnis auf der Timeline abgelesen werden kann. Somit konnte dieses Konzept so nicht umgesetzt werden und bedurfte einer Überarbeitung.

\section{Indikator-Timeline mit impliziter Strategie}

Parameter und Indikatoren: Bei der zweiten Iteration wird die Timeline optisch verändert dargestellt und dient weiterhin der Visualisierung des zentralen Indikators „Zeit bis letzter roter Patient von der Einsatzstelle abtransportiert wurde“. Im Vergleich zum vorherigen 
Konzept erfolgt bewusst keine parallele Visualisierung mehrerer Strategien (siehe Abb. 2). Die beiden Möglichkeiten der Parametereingabe von Iteration 1 (Dropdown der AAOEinsatzstichworte und Drag'n Drop) wurden beibehalten.

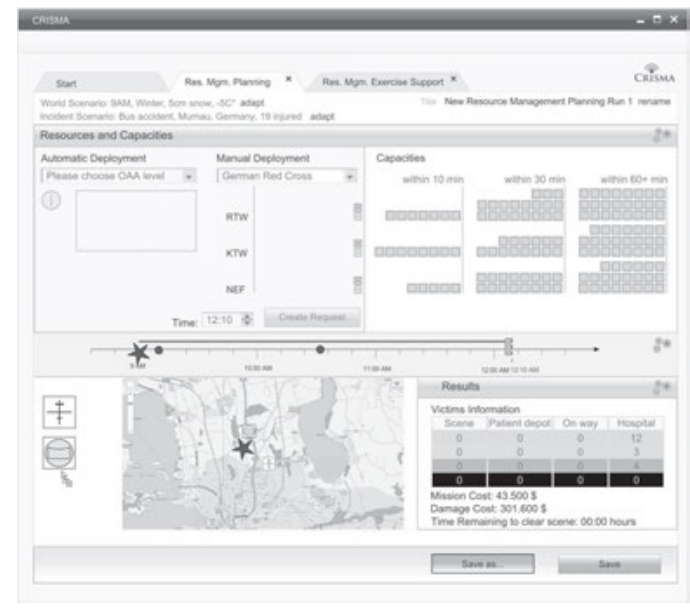

Abbildung 2: Konzept der Indikator-Timeline mit impliziter Strategie

Interaktion: Das Grundkonzept besteht hier darin, dass Änderungen der Ressourcenanforderungen oder die Verwendung eines bestimmten Einsatzabschnittes/einer taktischen Fläche (Patientenablage, Behandlungsplatz) eine Strategie implizieren und keine weiteren Angaben zu Einsatzabläufen notwendig sind. Dies hat sofortige Auswirkung auf das Modell und dessen Visualisierung in der Kartenansicht sowie der tabellarischen Patientenübersicht und der Indikator-Anzeige in der Timeline (rote Linie in Abb. 2). Die Ereignisleiste/der Slider kann innerhalb der Timeline vorwärts und rückwärts verschoben werden. Die Kartenansicht zeigt bei jeder Slider-Position die jeweils vor Ort vorhandenen Ressourcen zum jeweiligen Zeitpunkt in der Einsatzbewältigung. Diese Informationen geben dem professionellen Nutzer Auskunft darüber welche Einsatzstrategie am besten geeignet ist und unterstützen ihn so in der Entscheidungsfindung (Sautter et al. 2014a). Bei Fachanwendern bekannte taktische Zeichen dienen der Visualisierung von Fahrzeugen und Einsatzabschnitten.

Evaluation: Die Evaluierung fand, wie schon bei der ersten Iteration, in Form eines UserGuided Walkthroughs statt. Erkenntnis war, dass ein Indikator alleine nicht genug Aussagekraft besitzt, um den Benutzer in dessen Erkenntnisgewinn adäquat zu unterstützen. Außerdem erkennt der Nutzer trotz der visuellen Überarbeitung der Timeline nicht, dass das Hauptergebnis auf der Timeline abgelesen werden kann. Folglich sollten mehrere Indikatoren parallel visualisiert werden, um eine vergleichende Bewertung zu mehreren Erfolgskriterien der Einsatzbewältigung zu erzielen.

Technische Randbedingungen: Das Interaktionskonzept konnte im Projekt aus technischer Sicht nicht umgesetzt werden, da zur Umsetzung des hohen Grades an Interaktivität eine hohe Performanz und kurze Modelllaufzeit notwendig gewesen wäre. 


\section{$5 \quad$ Niedrige Interaktivität mit mehreren Indikatoren}

Parameter und Indikatoren: Die dritte Iteration stützt sich nicht mehr nur auf die Visualisierung eines einzelnen Indikators, sondern beinhaltet die Idee mehrere Indikatoren zu verwenden und den Grad an Interaktivität zu reduzieren. Dabei gibt es zwei separate Dialoge: In der Parametrisierung werden Szenarien mit hinterlegten Basisdaten zu Schadenslage und Ressourceninfrastruktur ausgewählt und vom Benutzer veränderliche Parameter eingegeben. Die Ergebnisanzeige dient nach Betätigung eines Start-Buttons der Visualisierung der Kernpunkte der Einsatzbewältigung und des Patienten-Outcomes mittels Diagrammen.
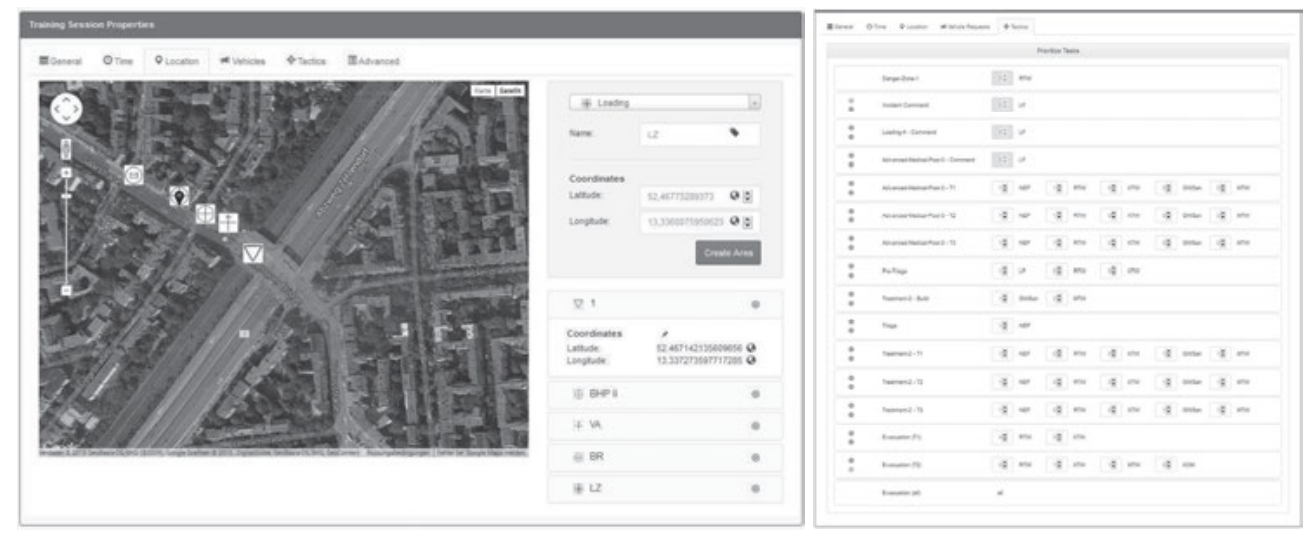

Abbildung 3: Parametrisierung der Einsatzabschnitte (a) und der zugehörigen einsatztaktischen Aufgaben (b)

Interaktion: Zunächst wird ein Schadensszenario gewählt (bspw. „Zugunglück in Berlin mit 213 Verletzten“). Die Anwendung lädt das Schadensszenario einschließlich der Rettungsinfrastruktur (Standorte Rettungswachen und Kliniken) sowie der lokalen AAO. Im nächsten Reiter „Locations“ (s. Abb. 3a) kann der Benutzer alle beabsichtigten Einsatzabschnitte definieren. Der nächste Reiter erlaubt die Definition der Ressourcenanforderungen im Zeitverlauf. Bspw. kann die Anforderung von vier Rettungstransportwagen (RTW) und zwei Löschfahrzeuge (LF) eine Minute nach dem Vorfall bereits im Dialog vorgegeben sein. Je nach Lagebild des ersteintreffenden Fahrzeuges und entsprechender Nachforderung und Einsatztaktik, kann der Benutzer bspw. eine frühe Ressourcenanforderung von Katastrophenschutzeinheiten nach 12 Minuten definieren. Im Reiter „Tactics“ besteht die Möglichkeit bestimmten einsatztaktischen Aufgaben Ressourcen zuzuordnen und diese zu priorisieren (siehe Abb. 3b). Im nächsten Schritt wird die Simulation gestartet, in dem der Button „Start Planning Session“ geklickt wird. Der Ergebnisanzeige (siehe Abb. 4) zeigt dann am oberen Bildschirmrand die benötigten Zeiten für die einzelnen Phasen der Einsatzbewältigung. Die Werte der Simulations-Indikatoren werden am rechten Bildschirmrand angezeigt. Die Zeiten, die Patienten einer Sichtungskategorie in einem bestimmten Zustand verblieben sind, werden in einem Balkendiagramm visualisiert. Das 
Beispiel in Abbildung 4 zeigt, dass der erste rote Patient die ersten präklinischen Maßnahmen ca. 25 Minuten nach dem Zwischenfall erhielt.

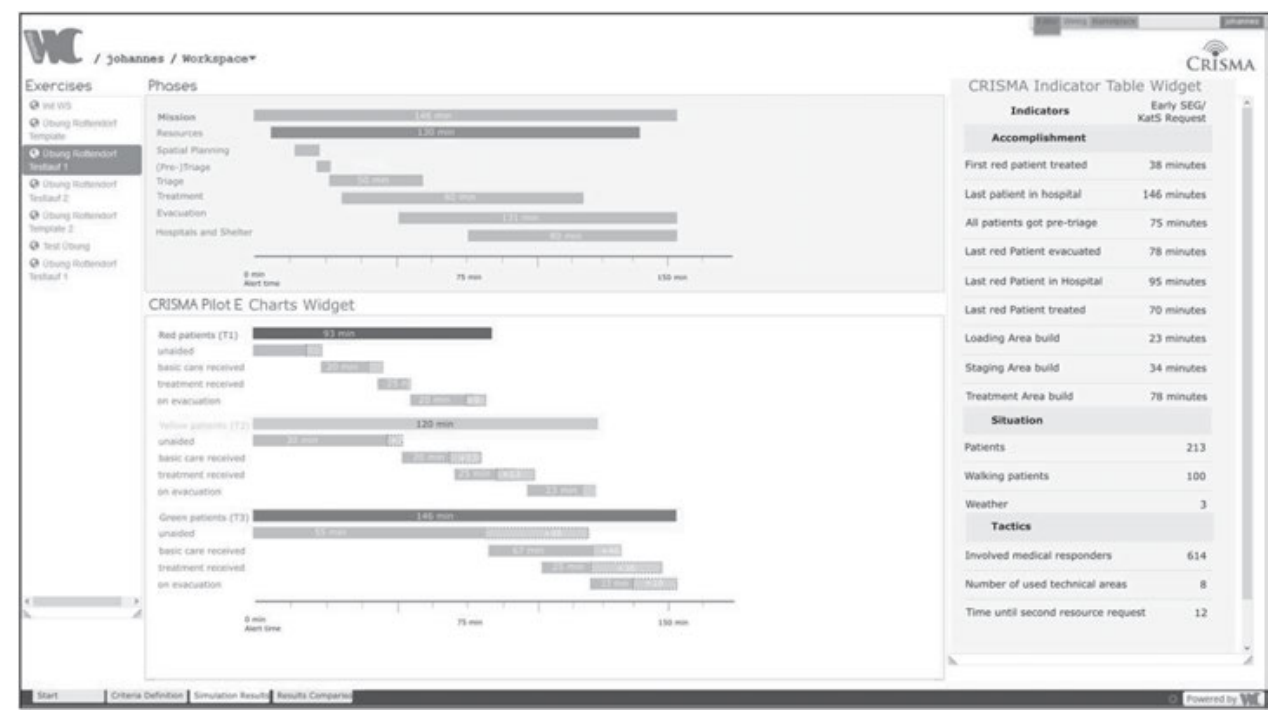

Abbildung 4: Ergebnisanzeige nach erfolgtem Simulationslauf

Evaluation: Neben Experten-Reviews konnten zur Validierung der Interaktionskonzepte zu Parametrisierung und Ergebnisanzeige auch Nutzertests genutzt werden, bei denen die Teilnehmer nach der „Think-Aloud-Methode“ ihre Eindrücke und Interpretationen laut mitteilen. Im Reiter „Location“ bereitete den Testpersonen das Setzen der Einsatzabschnitte erhebliche Probleme. Innerhalb des Reiters „Vehicle Requests“ bereitete den Testpersonen vor allem das Hinzufügen und Löschen von Fahrzeugen Probleme. Im Reiter „Tactics“ fällt auf, dass die Testpersonen nicht erkennen, dass die dort angezeigten einsatztaktischen Aufgaben aus den zuvor definierten Einsatzabschnitten abgeleitet sind.

Technische Randbedingungen: Zur Umsetzung wurde ein agentenbasiertes Simulationsmodell verwendet, das eine längere Laufzeit besitzt (mehrere Minuten).

\section{Zusammenfassung und Ausblick}

Wesentliche Aspekte zur Konzeption und Evaluation von Interaktionskonzepten, die durch Fachanwender bedient werden können, wurden in diesem Artikel beschrieben. Die Ergebnisse der Evaluation lassen darauf schließen, dass es noch Optimierungspotential im Hinblick auf Modellierung, Usability und Interaktivität gibt. Gewonnene Erkenntnisse von AnalyseSimulationsläufen können verwendet werden um Massenunfallszenarien mittels Feld- oder Kommandoübungen zu trainieren. Ferner können sie für eine Korrektur operativer Taktik- und Einsatzpläne und zur Erarbeitung lokal-spezifischer Handlungsempfehlungen für 
Führungskräfte entscheidend sein. Dies ist insofern besonders relevant, da in vielen Landkreisen - glücklicherweise - Erfahrungen aus Real-Einsätzen fehlen.

Ein zusätzliches Wunschfeature der Benutzer war es einen dynamische Patientenverlauf zu modellieren, was mangels Datengrundlage nicht möglich war. Mit einem technischperformanteren Modell könnte der Grad der Interaktion erhöht werden, so dass der Benutzer ähnlich den ersten beiden Konzepten explorativ vorgehen kann. Die Integration von bekannten „Gaming Features“ könnten dabei helfen die Anwendung nutzerfreundlicher zu gestalten.

\section{Danksagung}

Besonderer Dank gilt neben den geduldigen Führungskräften des DRK Matthias Max, Janina Hofer, Daniel Orlowski und Patrick Drews sowie unseren Projektpartnern Maria Egly, Peter Kutschera und Denis Havlik von AIT, Martin Scholl von cismet, Martin Sommer, Frank Jonat und Holger Bracker von Airbus Defence and Space sowie Kalev Rannat mit Team von der Tallinn University of Technology und Oren Deri von NICE. Das Projekt CRISMA wird vom Technischen Forschungszentrum Finnlands VTT geleitet und durch die EU kofinanziert (FP7/2007-2013, grant agreement no. 284552, www.crismaproject.eu).

\section{Literaturverzeichnis}

Brailsford, S. C., Harper, P. R., Patel, B., and Pitt, M. (2009). “An analysis of the academic literature on simulation and modelling in health care.” J Simulation 3 (3): 130-40. doi:10.1057/jos.2009.10.

Bundesamt für Bevölkerungsschutz und Katastrophenhilfe (BBK), ed. (2013). BBK-Glossar: “Ausgewählte zentrale Begriffe des Bevölkerungsschutzes". Stand/Auflage 02/2013 8. Bonn.

Carlson et al. (Hg.) (2005): “Discrete-event system simulation”. 4th ed. Upper Saddle River, NJ: Pearson Prentice Hall.

Dihé, P.; Denzer, R.; Polese, M.; Heikkilä, A.; Havlik, D.; Sautter, J.; Hell, T.; Schlobinski, S.; Zuccaro, G. und Engelbach, W. (2013). "An architecture for integrated crisis management simulation.”. Proceedings of the 20th International Congress on Modelling and Simulation, Adelaide, Australia, 1-6 December 2013.

Deutsches Rotes Kreuz. "Zukünftige Schwerpunkte und Forschungsbedarfe im Deutschen Roten Kreuz", Newsletter - Neues aus der Sicherheitsforschung, 2012.

Railsback, Steven F.; Grimm, Volker (Hg.) (2012): “Agent-based and individual-based modeling. “ A practical introduction. Princeton: Princeton University Press.

Sautter, J.; Hofer, J.; Wirth, S.; Engelbach, W.; Max, M.; Tenso, T. und Bracker, H. (2014a). "Localspecific resource planning for mass casualty incidents." Proceedings of the 11th International ISCRAM Conference - University Park, Pennsylvania, USA, May 2014.

Sautter, J.; Habermann, M.; Frings, S.; Schneider, F.; Schneider, B. und Bracker, H. (2014b). “Übungsunterstützung für Einsatztrainings des Massenanfalls von Verletzten (MANV)." Tagung der Gesellschaft für Informatik, 22.-26. September 2014 in Stuttgart, Deutschland Bonn: Köllen, 2014 (GI-Edition - Lecture Notes in Informatics (LNI) - Proceedings 232). 
Tisue, S.; Wilensky, U. (2004): NetLogo: "Design and implementation of a multi-agent modeling environment". In: Design and implementation of a multi-agent modeling environment. Proceedings of the Agent 2004 Conference on Social Dynamics: Interaction, Reflexivity and Emergence, Chicago, IL. 
